\title{
Theory and Practice of Permutation Entropy
}

\author{
Karsten Keller, Inga Stolz \\ Institute of Mathematics \\ University of Lübeck
}

4th International Electronic Conference on Entropy and Its Applications, 21 November - 1 December 2017 


\title{
Contents
}

\author{
(1) Motivation
}

(2) Context

(3) From KS entropy to Permutation entropy

(4) Conditional viewpoint

(5) Practical aspects 


\section{Motivation}


- Entropies and entropy-like quantities are playing an increasing role in data analysis, in the contexts of dynamical systems and of stochastic processes.

- Various applications of entropies are given, for example, in the analysis of physiological time series.

- Central classical concepts: Approximate entropy, Sample entropy, and variants.

- Interesting relatively new concepts: Permutation entropies and variants based on the ordinal structure of time series and systems behind them.

- Important: good theoretical understanding of those concepts and their relationship to other entropies, and their adequate application to the analysis of data. 
2. Context 


\section{Modeling (one observable, simplest setting)}

time series $\left(\mathrm{x}_{\mathrm{t}}\right)_{\mathrm{t}=0}^{\mathrm{N}-1}=\left(\mathrm{x}_{0}, \mathrm{x}_{1}, \mathrm{x}_{2}, \ldots, \mathrm{x}_{\mathrm{N}-1}\right)$ assumed as values measured on the orbit of a dynamical system

- $(\Omega, \mathcal{A}, \mu, \mathrm{T}) \mu$-preserving dynamical system

- X real-valued random variable on $\Omega$

- observable $X$ provides 'outreading' stochastic process $\left(X \circ T^{\circ t}\right)_{t=0}^{\infty}$ with realizations $\left(\mathrm{X} \circ \mathrm{T}^{\circ \mathrm{t}}(\omega)\right)_{\mathrm{t}=0}^{\infty} ; \omega \in \Omega$

\section{assumptions:}

- Ergodicity: realizations represent distribution of $X$

- no information loss by the measuring process with respect to $X$ ('separation' of orbits), natural' 


\section{Ordinal patterns}

\section{Definition}

$\left(x_{0}, x_{1}, \ldots, x_{d}\right) \in \mathbb{R}^{d+1}$ has the (ordinal) d-pattern $\pi=\left(r_{0}, r_{1}, \ldots, r_{d}\right) \in \Pi_{d}$ if

$$
\begin{aligned}
x_{r_{0}} & \geq x_{r_{1}} \geq \ldots \geq x_{r_{d}}, \text { and } \\
r_{1-1} & >r_{1} \text { in the case } x_{r_{1-1}}=x_{r_{1}} .
\end{aligned}
$$
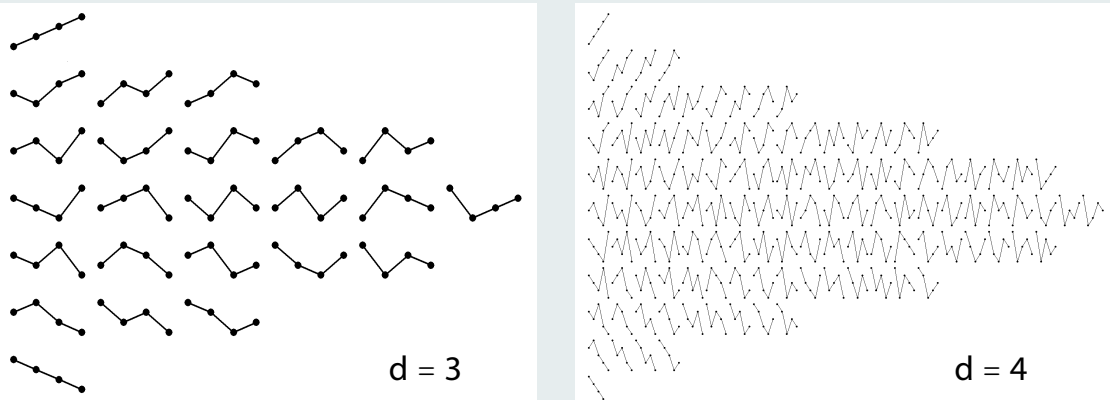
Relative frequencies / probabilities of ordinal d-pattern words

\section{Definition}

- relative frequency of d-patt. word $\left(\pi_{1}, \pi_{2}, \ldots, \pi_{k}\right) \in \Pi_{d}^{k}$ in time series $\left(x_{t}\right)_{t=0}^{N-1}$ :

$$
\begin{array}{r}
\mathrm{p}_{\left(\pi_{1}, \pi_{2}, \ldots, \pi_{\mathrm{k}}\right)}:=\frac{1}{\mathrm{~N}-\mathrm{d}-\mathrm{k}+1} \#\{\mathrm{~s} \in\{0, \ldots, \mathrm{N}-\mathrm{d}-\mathrm{k}\} \mid \text { for all } \mathrm{i}=1, \ldots, \mathrm{k} \\
\left.\left(\mathrm{x}_{\mathrm{s}+\mathrm{i}-1}, \mathrm{x}_{\mathrm{s}+\mathrm{i}} \ldots, \mathrm{x}_{\mathrm{s}+\mathrm{i}-1+\mathrm{d}}\right) \text { has ordinal pattern } \pi_{\mathrm{i}}\right\}
\end{array}
$$

- probability of d-pattern word $\left(\pi_{1}, \pi_{2}, \ldots, \pi_{\mathrm{k}}\right) \in \Pi_{\mathrm{d}}^{\mathrm{k}}$ for $(\Omega, \mathcal{A}, \mu, \mathrm{T}, \mathrm{X})$ :

$$
\begin{aligned}
\mathrm{P}_{\left(\pi_{1}, \pi_{2}, \ldots, \pi_{\mathrm{k}}\right)} & :=\mu(\{\omega \in \Omega \mid \text { for all } \mathrm{i}=1, \ldots, \mathrm{k} \\
\mathrm{X} & \left.\left.\circ \mathrm{T}^{\circ \mathrm{i}-1}(\omega), \mathrm{X} \circ \mathrm{T}^{\circ \mathrm{i}}(\omega), \ldots, \mathrm{X} \circ \mathrm{T}^{\circ \mathrm{d}}(\omega) \text { has ordinal pattern } \pi_{\mathrm{i}}\right\}\right)
\end{aligned}
$$

- $\mathrm{p}_{\left(\pi_{1}, \pi_{2}, \ldots, \pi_{\mathrm{k}}\right)}$ estimates $\mathrm{P}_{\left(\pi_{1}, \pi_{2}, \ldots, \pi_{\mathrm{k}}\right)}$ 


\section{From KS entropy} to permutation entropy 


\section{Entropies (I)}

Kolmogorov-Sinai entropy (KS entropy) is central theoretical complexity measure for dynamical systems, but not easy to determinate and to estimate.

ordinal approach:

- entropy of d-pattern-k-words:

$$
\mathrm{H}(\mathrm{d}, \mathrm{k}):=-\sum_{\left(\pi_{1}, \ldots, \pi_{\mathrm{k}}\right) \in \Pi_{\mathrm{d}}^{\mathrm{k}}} \mathrm{P}_{\left(\pi_{1}, \ldots, \pi_{\mathrm{k}}\right)} \ln \mathrm{P}_{\left(\pi_{1}, \ldots, \pi_{\mathrm{k}}\right)}
$$

- empirical entropy of d-pattern-k-words:

$$
\mathrm{h}(\mathrm{d}, \mathrm{k}):=-\sum_{\left(\pi_{1}, \ldots, \pi_{\mathrm{k}}\right) \in \Pi_{\mathrm{d}}^{\mathrm{k}}} \mathrm{p}_{\left(\pi_{1}, \ldots, \pi_{\mathrm{k}}\right)} \ln \mathrm{p}_{\left(\pi_{1}, \ldots, \pi_{\mathrm{k}}\right)}
$$

- $h(d, k)$ estimates $H(d, k)$

- $\mathrm{H}(\mathrm{d}, 0):=0$ 


\section{Entropies (II)}

- entropy rate of $d$-patterns:

$$
\text { EntroRate }(d):=\lim _{k \rightarrow \infty}(\downarrow) \frac{H(d, k)}{k}=\lim _{k \rightarrow \infty}(\downarrow)(H(d, k+1)-H(d, k))
$$

\section{Theorem}

$$
\text { KS entropy }=\lim (\uparrow)_{d \rightarrow \infty} \text { EntroRate }(d) \leq \text { Permutation entropy }{ }^{2}
$$

${ }^{2}$ Antoniouk, K., Maksymenko, Discrete Contin. Dyn. Syst. A 34 (2014); K., Maksymenko,Stolz, Discrete Contin. Dyn. Syst. B 20 (2015);

- only one limit for Permutation entropy3:

$$
\text { Permutation entropy }=\limsup _{d \rightarrow \infty} \frac{H(d, 1)}{d}
$$

(better: $\left.\liminf _{d \rightarrow \infty} \frac{H(d, 1)}{d}\right)$ 


\section{Entropies (III)}

\section{Remark}

KS entropy = Permutation entropy for a special class of dynamical systems (piecewise monotone interval maps) and X identity ${ }^{4}$

${ }^{4}$ Bandt, G. Keller, Pompe, Nonlinearity 15 (2002) 


\section{General ordinal view on KS entropy}

relevant entropies:

$\begin{array}{llllllll}\mathrm{H}(1,1) & \mathrm{H}(1,2) & \mathrm{H}(1,3) & \mathrm{H}(1,4) & \mathrm{H}(1,4) & \mathrm{H}(1,5) & \mathrm{H}(1,6) & \ldots \\ \mathrm{H}(2,1) & \mathrm{H}(2,2) & \mathrm{H}(2,3) & \mathrm{H}(2,4) & \mathrm{H}(2,4) & \mathrm{H}(2,5) & \mathrm{H}(2,6) & \ldots \\ \mathrm{H}(3,1) & \mathrm{H}(3,2) & \mathrm{H}(3,3) & \mathrm{H}(3,4) & \mathrm{H}(3,4) & \mathrm{H}(3,5) & \mathrm{H}(3,6) & \ldots \\ \mathrm{H}(4,1) & \mathrm{H}(4,2) & \mathrm{H}(4,3) & \mathrm{H}(4,4) & \mathrm{H}(4,4) & \mathrm{H}(4,5) & \mathrm{H}(4,6) & \cdots\end{array}$

right weighting:

$H(1,1) \quad H(1,2) / 2 \quad H(1,3) / 3 \quad H(1,4) / 4 \quad H(1,5) / 5 \quad \cdots \quad \searrow \quad$ EntroRate(1) $H(2,1) \quad H(2,2) / 2 \quad H(2,3) / 3 \quad H(2,4) / 4 \quad H(2,5) / 5 \quad \cdots \quad \searrow \quad$ EntroRate(2) $\begin{array}{llllllll}H(3,1) & H(3,2) / 2 & H(3,3) / 3 & H(3,4) / 4 & H(3,5) / 5 & \cdots & \searrow & \text { EntroRate(3) }\end{array}$ $\begin{array}{llllllll}H(4,1) & H(4,2) / 2 & H(4,3) / 3 & H(4,4) / 4 & H(4,5) / 5 & \cdots & \searrow & \text { EntroRate(4) }\end{array}$ 


\section{Generalizations}

- generalization to finitely (infinitely) many observables $\mathrm{X}_{1}, \mathrm{X}_{2}, \ldots, \mathrm{X}_{\mathrm{n}}(, \ldots)$

- generalization to many non-ergodic cases (ergodic decomposition)

- generalization to new two-dimensionel basic symbolization schemes ${ }^{5}$
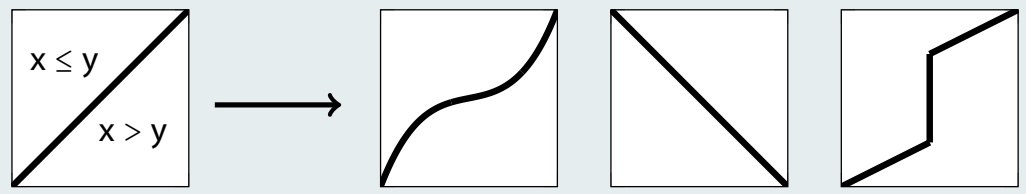
4. Conditional viewpoint 


\section{'Conditional' ordinal view on KS entropy}

$\begin{array}{llllll}\mathrm{H}(1,2)-\mathrm{H}(1,1) & \mathrm{H}(1,3)-\mathrm{H}(1,2) & \mathrm{H}(1,4)-\mathrm{H}(1,3) & \cdots & \searrow & \text { EntroRate(1) } \\ \mathrm{H}(2,2)-\mathrm{H}(2,1) & \mathrm{H}(2,3)-\mathrm{H}(2,2) & \mathrm{H}(2,4)-\mathrm{H}(2,3) & \cdots & \searrow & \text { EntroRate(2) } \\ \mathrm{H}(3,2)-\mathrm{H}(3,1) & \mathrm{H}(3,3)-\mathrm{H}(3,2) & \mathrm{H}(3,4)-\mathrm{H}(3,3) & \cdots & \searrow & \text { EntroRate(3) } \\ \mathrm{H}(4,2)-\mathrm{H}(4,1) & \mathrm{H}(4,3)-\mathrm{H}(4,2) & \mathrm{H}(4,4)-\mathrm{H}(4,3) & \cdots & \searrow & \text { EntroRate(4) }\end{array}$

- For all natural numbers $\mathrm{k}$ it holds

$$
H(d, k)-H(d, k-1) \leq \frac{1}{k} \sum_{i=1}^{k}(H(d, i)-H(d, i-1))=\frac{H(d, k)}{k}
$$

\section{Lemma}

For each sequence $\left(k_{d}\right)_{d=1}^{\infty}$ of natural numbers it holds

KS entropy $\leq \liminf _{d \rightarrow \infty}\left(H\left(d, k_{d}+1\right)-H\left(d, k_{d}\right)\right) \leq \liminf _{d \rightarrow \infty} \frac{H\left(d, k_{d}\right)}{k_{d}}$ 


\section{Conditional entropy of ordinal patterns ${ }^{6}$}

- special case: $k_{d}=1$ for all $d \in \mathbb{N}$ :

\section{Definition}

\section{conditional entropy of ordinal patterns}

$$
\liminf _{d \rightarrow \infty} H(d, 2)-H(d, 1)
$$

- interpretation: uncertainty of next type of ordinal pattern of high order given one such type

- also interesting:

$$
\liminf _{d \rightarrow \infty} H(d, k+1)-H(d, k)
$$

for $\mathrm{k}=2,3,4, \ldots$ 


\section{Conditional entropy better than PE?}

assuming existence of

$$
\lim _{d \rightarrow \infty}(H(d+1,1)-H(d, 1)),
$$

which looks natural, by the Stolz-Cesàro theorem with $\mathrm{H}(\mathrm{d}, 0):=0$ it holds

$$
\begin{aligned}
\text { KS entropy } & \leq \liminf _{d \rightarrow \infty}(H(d, 2)-H(d, 1)) \\
& =\text { conditional entropy of ordinal patterns } \\
& \leq \lim _{d \rightarrow \infty}(H(d+1,1)-H(d, 1)) \\
& =\lim _{d \rightarrow \infty}(H(d, 1)-H(d-1,1)) \\
& =\lim _{d \rightarrow \infty} \frac{1}{d} \sum_{i=1}^{d}(H(i, 1)-H(i-1,1)) \\
& =\liminf _{d \rightarrow \infty} \frac{1}{d} H(d, 1)=\text { Permutation entropy }
\end{aligned}
$$




\section{Logistic family and permutation entropies}

- $x \in[0,1] \mapsto r x(1-x)$ for different $r \in[0,4]$

- for almost all $r \in[0,4]$ the KS entropy either coincides with the Lyapunov exponent if it is positive or is equal to zero otherwise (Pesin's formula, 'natural' RB-measure)

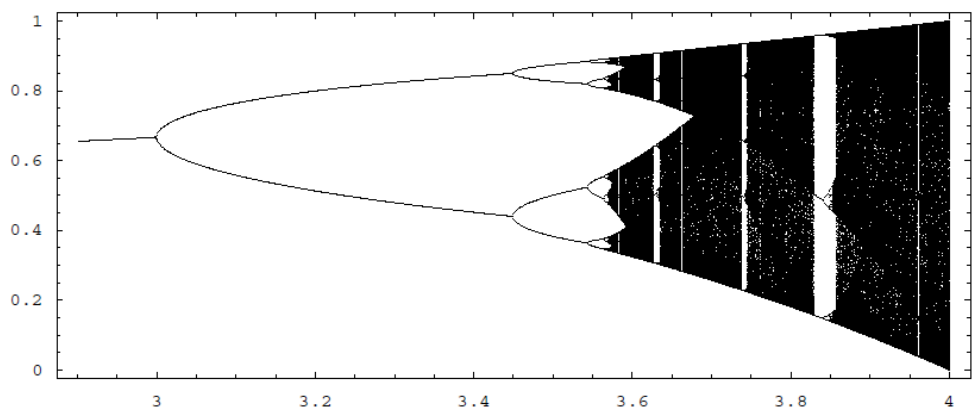




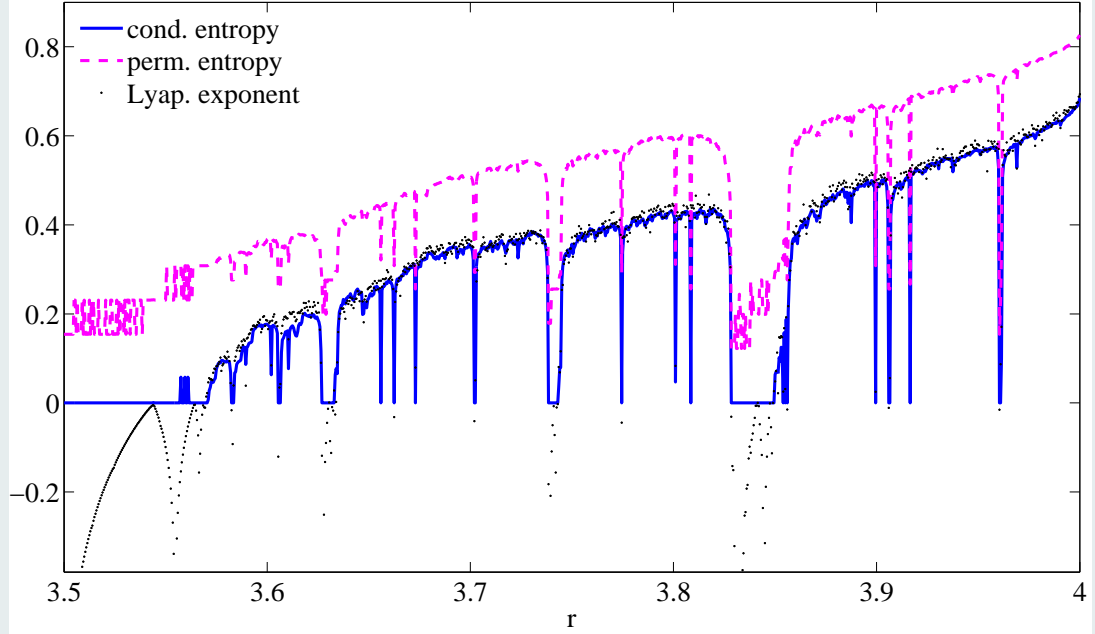

Lyapunov exponent, empirical and conditional Permutation entropy ( $d=9$ ) 


\section{Practical aspects}




\section{Asymptotics versus statistics ${ }^{7}$}

$$
\Omega=[0,1], T(\omega)=4 \omega(1-\omega), X=\text { id }
$$
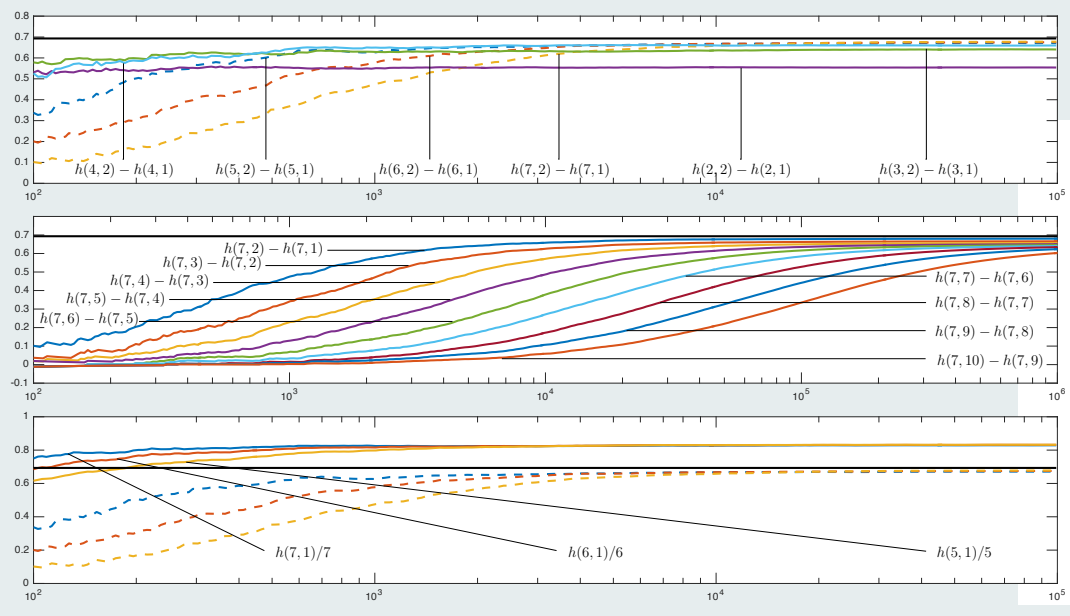

${ }^{7}$ Keller, Mangold, Stolz, Werner Entropy 19 (2017) 


\section{Combining entropies, delays}

example: EEG recordings from the Bonn EEG Database ${ }^{8}$
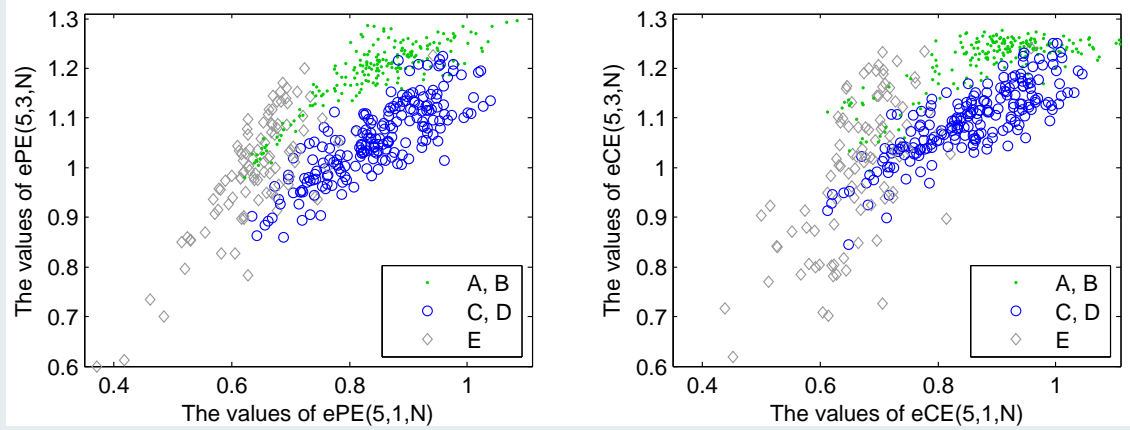

empirical Permutation entropy versus empirical Permutation entropy/ conditional Permutation entropy versus conditional Permutation entropy, also other entropies 


\section{Variants of Permutation entropy}

\section{Reinventing metric information}

- 'Flat' ordinal patterns, i.e. ordinal patterns coming from vectors of low variability, are very sensitive with respect to noise.

$\Rightarrow$ robust Permutation entropy ${ }^{9}$ excluding 'flat' ordinal patterns

$\Rightarrow$ weighted Permutation entropy ${ }^{10}$ : weighting ordinal patterns by variance of vectors behind

- theoretically not well understood

Generalization of conditional approach by Armand Eyebe Fouda, Koepf, Jacquir ${ }^{11}$

9 Keller, Unakafov, Unakafova, Entropy 16 (2014)

${ }^{10}$ Fadlallah, Chen, Keil, Prìncipe, Phys. Rev. E 87 (2013)

${ }^{11}$ Communications in Nonlinear Sci. and Num. Simulat. 46 (2017), 103-115 


\section{Further aspects}

- generally: compromise between theoretical requirements and practical possibilities necessary

- requires better theoretical understanding of measures

- results in standardizations which have to be communicated and commonly used

- standards have to be (automatically) related to kind and size of data

- finding best measures also needs application of machine learning 


\section{Thank you!}

